\title{
Colonic volvulus in the spinal cord injured patient
}

\author{
D Fenton-Lee FRACS ${ }^{1}$ B W Yeo FRACS FRCS, ${ }^{1}$ R F Jones FRACS FRACP \\ FACRM ${ }^{2}$ S Engel DPRM FACRM ${ }^{2}$
}

Departments of ${ }^{1}$ Surgery and ${ }^{2}$ Rehabilitation Medicine, Prince Henry Hospital, New South Wales, Australia.

At the Prince Henry Hospital between 1970 and 1987, 8 cases of colonic volvulus were diagnosed in spinal cord injured (SCI) patients. This represents an incidence of $2.6 \%$ of SCI patients being treated at the hospital. The aetiology of volvulus is multifactorial and in these patients may be related to: neurological deficit; constipation as a result of immobility; and medications used for controlling muscle spasm, bladder and bowel function.

Symptoms and signs are modified by the neurological deficit and awareness of the altered presentation will prevent delay and missed diagnoses. The effect of spinal cord injury and drugs on colonic motility needs further evaluation.

Keywords: volvulus; sigmoid volvulus; spinal cord injury; intestinal obstruction; colonic diseases.

\section{Introduction}

Rokitansky is attributed with the first description of colonic volvulus in 1836 but long before this the ancient Egyptians, as well as Hippocrates, had recognized the condition. ${ }^{1}$ In $1859 \mathrm{Mr}$ Gay successfully reduced a sigmoid volvulus in a postmortem patient by inserting a rectal tube, a method of treatment not dissimilar to that advocated by Hippocrates. ${ }^{2}$

In the twentieth century Treves, Lecene, Senn, Von Eiselsberg and Moynihan were able to demonstrate that earlier surgical intervention in the management of sigmoid volvulus resulted in lower mortality. ${ }^{3-7}$ The basis of modern day management evolved in 1947 when Bruusgard demonstrated an even greater reduction in mortality when sigmoidoscopic detorsion and decompression of the volvulus was attempted prior to definitive surgery. ${ }^{8}$

Colonic volvulus is relatively uncommon despite being the third commonest cause of mechanical large bowel obstruction in western countries. Two (SCI) patients with colonic volvulus presented to the Prince

Correspondence: $\mathrm{Mr} \mathrm{D}$ Fenton-Lee, Clinical Lecturer, University Department of Surgery, Glasgow Royal Infirmary, Glasgow, UK.
Henry Hospital during 1987 which prompted a review of this condition in SCI patients.

\section{Methods}

We reviewed the case notes of all patients with colonic volvulus between 1970 and 1987. Particular note was made of possible aetiological factors, clinical findings and management of the condition.

\section{Clinical features}

Colonic volvulus was diagnosed in $8 \mathrm{SCI}$ and 57 non spinal cord injured (NSCI) patients at the Prince Henry Hospital between 1970 and 1987. This represents an incidence of $2.6 \%$ for the SCI patients based on the number being treated at the hospital during this period. A summary of clinical details can be seen in Table I.

The symptoms of bowel obstructionabdominal pain, distension, obstipation and vomiting - were less severe and chronic in the SCI group compared with the more typical acute and severe presentation in the NSCI group. The time from spinal cord injury to onset of symptoms compatible with colonic volvulus varied from 1 to 20 years. There was no correlation between the level 
Table I Summary of clinical features in spinal cord injured and normal patients with colonic volvulus

\begin{tabular}{lcc}
\hline & SCI patients $(n=8)$ & Non SCI patients $(n=57)$ \\
\hline Mean age presentation (years) & 45.5 & 63 \\
Sex & $\mathrm{M}>\mathrm{F}$ & $\mathrm{M}>\mathrm{F}$ \\
Presentation & Chronic & Acute \\
Site volvulus & Sigmoid $>$ Caecal & Sigmoid $>$ Caecal \\
Mortality & $12 \%$ & $9 \%$ \\
\hline
\end{tabular}

of injury or completeness of injury and the time to presentation.

Examination of the SCI patients revealed abdominal distension with no palpable masses. Four patients had tenderness which was poorly localised: 6 out of 8 patients had obstructive bowel sounds; the 2 with absent bowel sounds had gangrene of the bowel. Patients who had sigmoid volvulus all had empty rectums on digital examination. All SCI patients had been prescribed laxatives which contained senna or cascara. Patients had at some time been prescribed baclofen, ganglion blockers, anticholinergics or psychotropic drugs.

Volvulus was diagnosed by plain abdominal xray in 4 patients and barium enema in 2. A plain abdominal xray demonstrating a sigmoid volvulus can be seen in Figure 1 and caecal volvulus in Figure 2. Features pathogenmonic for each type of volvulus can be seen in Tables II and III.

Caecopexy was performed on the 2 patients with caecal volvulus; subsequently one had a right hemicolectomy for recurrence and the other was lost to follow up. The 6 patients with sigmoid volvulus were all decompressed and detorted by sigmoidoscopy and insertion of a rectal tube (Fig 3). These patients subsequently had elective sigmoid resection and one patient died due to an anastomotic leak.

\section{Discussion}

Colonic volvulus is uncommon and any one clinician would expect to see only a handful of cases during his/her career. The condition occurs more commonly in the sigmoid colon than in the caecum with a ratio of $9: 1 .{ }^{9}$ In our community it most commonly affects

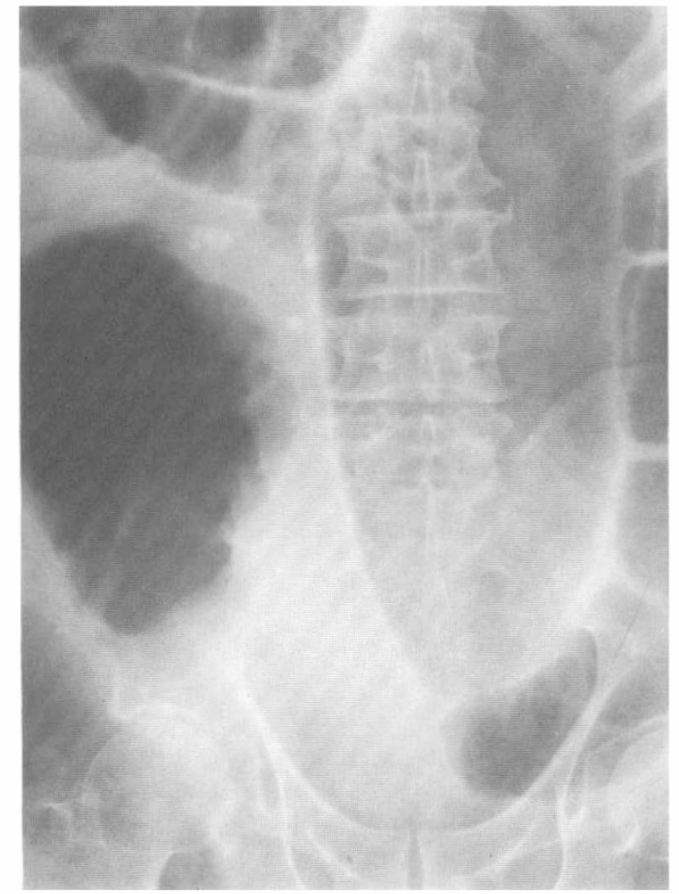

Figure 1 Sigmoid volvulus showing 'bent inner tube' appearance with 'bird beak deformity' and evidence of other distended large bowel.

institutionalised patients, psychiatric patients, the elderly and the infirm. ${ }^{10-12}$ The aetiology is unknown but is thought to be multifactorial. Factors thought to be important are constipation, immobility, poor diet, inattention to bowel habit, a high fibre diet and the narrow android pelvis which is thought to account for the higher incidence in males. ${ }^{9,13}$

In SCI patients immobility and constipation may be factors which predispose to volvulus formation. We wondered whether the neurological deficit and medications 


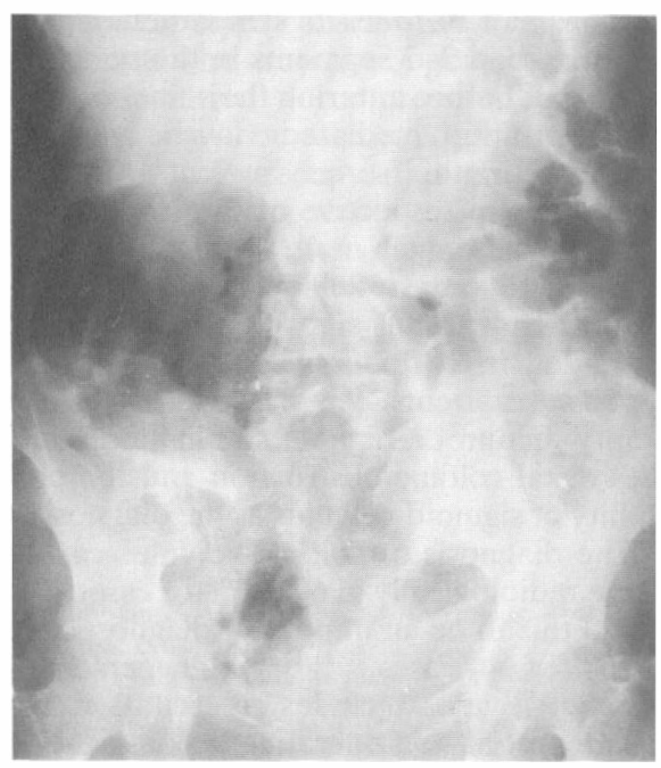

Figure 2 Caecal volvulus showing typical distended 'kidney shape' caecum in ectopic position.

used in these patients were possible aetiological factors. However we could not find an association between the level of SCI or a temporal relationship with the occurrence of volvulus. The younger age of presentation in the SCI patients is probably related to the younger age of the group in general. Therefore the spinal cord injury in itself does not

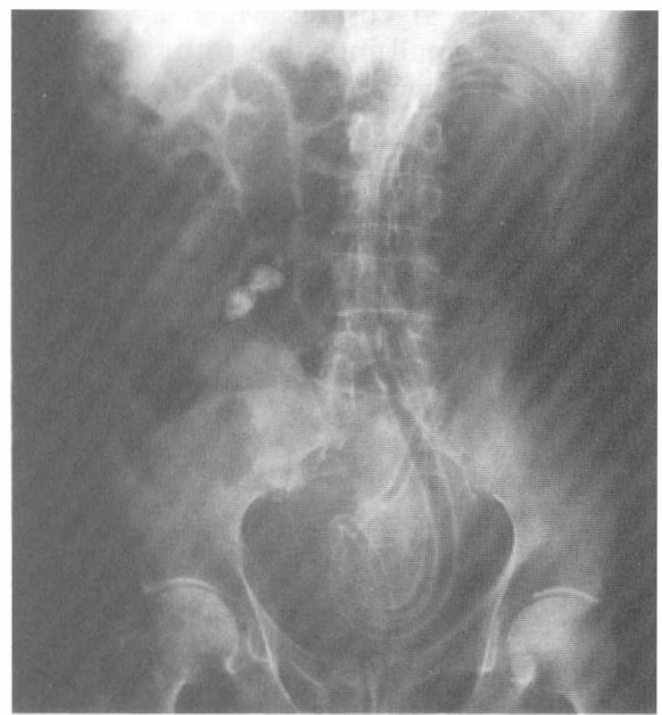

Figure 3 Sigmoid volvulus with rectal tube in situ following sigmoidoscopic decompression.

appear to be a major predisposing factor for colonic volvulus. The long term effects of the medications used to control muscle spasm and regulate bowel and bladder habit on smooth muscle activity is not widely known. ${ }^{14}$

The SCI patients had been on long term laxative preparations containing senna or cascara. These are ingested as inactive

Table II Radiological features of sigmoid volvulus

Bent inner tube appearance with apex pointing down to the left iliac fossa; two fluid levels at its base

Remainder of the large bowel dilated but not to the same extent

Usually an absence of gas in the rectum

'Bird beak' deformity which can be seen on a plain abdominal xray, though can be seen more prominently on a barium enema

Table III Radiological features of caecal volvulus

Greatly distended caecum in an ectopic position, most commonly in the left upper quadrant or right lower quadrant

Distended small intestine often located to the right of the caecum producing a 'kidney shape'

Visualisation of the ileocaecal valve on the right side of the caecum

Mucosal folds producing a spiral at the point of obstruction 
anthraquinone glycosides which bypass the stomach and small intestine to be activated by colonic bacteria. The activated anthraquinone derivative appears to act by contact stimulation of the submucosal nerve plexuses which results in the initiation of coordinated peristalsis. ${ }^{14,15}$ The prolonged usage of these laxatives may cause neuronal degeneration and axonal dropout in the myenteric plexuses and produce colonic dilatation, a predisposing factor in volvulus formation. ${ }^{16}$

There is circumstantial evidence that psychotropic drugs such as the phenothiazines and amitriptyline also play an aetiological role. They are frequently prescribed for SCI patients and are known to produce paralytic ileus, constipation and megacolon with prolonged usage. ${ }^{17,18}$ These drugs are also commonly used in patients residing in mental institutions and nursing homes who comprise a significant proportion of patients documented with colonic volvulus. ${ }^{10-12,19,20}$

Muscle relaxants such as Lioresal (baclofen) are used in SCI patients for the relief of skeletal muscle spasm. The pharmacology of Lioresal though poorly understood is thought to act at the presynaptic level producing inhibition at both polysynaptic and monosynaptic transmission. ${ }^{14}$ There are no reported studies of its action on human colonic smooth muscle and it is variable in animal studies. The other drugs commonly used in this group of patients such as the anticholinergic, cholinergic and ganglion blockers may have adverse effects on smooth muscle function with prolonged use.

The delay in diagnosis of up to 3 days in some of our cases may be due to lack of suspicion of the diagnosis and difficulty in interpreting the clinical findings. The clinical findings of bowel obstruction are similar to faecal impaction or ileus secondary to urinary tract infection ${ }^{21-23}$ and masking of symptoms and signs of the acute abdomen occur due to the neurological deficit. ${ }^{23-25}$ For instance abdominal pain was absent in 2 cases and when present was poorly localised. This is thought to occur with lesions above T4 (high lesions) as a result of interrupted sympathetic pain fibres. ${ }^{24,25}$ The ability to localise pain despite having high lesions may be due to sympathetic fibres which ascend 2-3 segments in the sympathetic trunk before entering the spinal cord or pain sensation mediated via the vagus. ${ }^{24}$ However in this series, patients localised pain poorly irrespective of the level of the lesion.

Autonomic dysreflexia can be an important early warning sign of intraabdominal pathology in SCI patients and occurred in 2 of our 8 patients. ${ }^{22-25}$ The finding of an empty rectum on digital examination indicates distal colonic obstruction and the possibility of sigmoid volvulus as the diagnosis.

The diagnosis of colonic volvulus can be made radiologically in $60-90 \%$ of cases. We found this to be an important diagnostic aid in $75 \%$ of our cases. ${ }^{10,11,13,26}$ Management of colonic volvulus includes resuscitation and an attempt at non operative decompression and derotation. This is followed at a later date by definitive surgery in those patients fit for surgery. Reduction of a sigmoid volvulus is best achieved with a flexible fibre optic sigmoidoscope and a rectal tube is left in situ for a minimum of 3 days to prevent early recurrence. ${ }^{8,26,27}$ Gangrene of the bowel is associated with difficulty in reducing the volvulus, the presence of rectal bleeding or visualisation of non viable rectal mucosa. ${ }^{27}$ In these cases excessive force in the passage of the flatus tube or sigmoidoscope (rigid or flexible) may result in perforation of the bowel and require operative intervention to reduce the volvulus. The choice of operation will depend upon seveal factors such as the presence of necrotic bowel, perforation, peritonitis and the fitness of the patient. Sigmoid resection with primary anastomosis is the definitive operation; however in an emergency situation a Hartmann's procedure or exteriorisation of the bowel may be more appropriate. ${ }^{27}$ In this series of patients we had one death ( $12 \%$ mortality rate) following elective sigmoid resection due to an anastomotic leak. In reviewing the literature we find that patients treated by sigmoidoscopic reduction without definitive surgery have a recurrence rate of up to $42 \%$ and a mortality rate of $10.7 \% .{ }^{27}$ The best reported mortality rate for patients undergoing elective sigmoid resection is $8.5 \% .{ }^{27}$ There is therefore a 
case for repeated conservative management in patients who are a poor operative risk.

Nonoperative decompression of caecal volvulus may be achieved with barium enema or colonoscopy, though both techniques do not prevent early recurrence. Caecal volvulus is often diagnosed at laparotomy and the definitive operation is right hemicolectomy. ${ }^{28}$ Significant morbidity and recurrence are associated with the operations of caecopexy or caecostomy and are therefore not recommended. ${ }^{28}$

\section{Conclusion}

Colonic volvulus is generally uncommon though awareness of the altered presentation in SCI patients will prevent delay and misdiagnoses.

The effect of spinal cord injury and commonly used drugs on colonic motility needs further evaluation.

Definitive surgical management is safe and fit patients should be considered for surgery.

\section{References}

1 Drabkin IE (1950) Caelius Aurelianus: On Acute Diseases and on Chronic Diseases. University of Chicago Press, Chicago: $389-407$.

2 Gay J (1859) Fatal obstruction from twisting of the mesocolon. Trans Pathol Soc London 10: 153-154.

3 Treves F (1884) Intestinal obstruction, its varieties with their pathology, diagnosis and treatment. Henry C Lea and Company, Philadelphia.

4 Lecene P (1910) Contribution a l'étude des volvulus du gross intestin. Rev Chir 41: 21-44.

5 Senn N (1889) The surgical treatment of volvulus. Med News 55: 590-598.

6 Von Eiselberg F (1889) Zur Radicaloperation des Volvulus und der Invagination durch die Resection. Dtsch Med Wochenschr 25: 805-808.

7 Moynihan BG (1905) Abdominal Operations. W B Saunders, Philadelphia.

8 Bruusgard C (1947) Volvulus of the sigmoid colon and its treatment. Surgery 22: 466-478.

9 Ballantyne GH (1982) Review of sigmoid volvulus: clinical pattern and pathogenesis. Dis Colon Rectum 25: 823-830.

10 Arnold GJ, Nance FC (1973) Volvulus of the sigmoid colon. Ann Surg 177: 527-537.

11 Hines JR, Geurkink RE, Bass RT (1967) Recurrence and mortality rates in sigmoid volvulus. Surg Gynecol Obstet 124: 567-570.

12 Hildebrand HD, Wilson JW (1967) Volvulus of the sigmoid with special reference to the role of ganglion deficiency as an aetiological factor. Can J Surg 10: 449-453.

13 Shepherd JJ (1968) Treatment of volvulus of sigmoid colon: a review of 425 cases. Br Med J 1: 280-283.

14 Martindale W (1989) The Extra Pharmacopoeia 29th ed. The Pharmaceutical Press London: 1105-1107, 1081.

15 Hardcastle JD, Wilkins JL (1970) The action of sennosides and related compounds on human colon and rectum. Gut 11: 1038-1042.

16 Smith B (1970) Disorders of the myenteric plexus. Gut 11: 271-274.

17 Warnes H, Lehmann HE, Ban TA (1967) Adynamyic ileus during psychoactive medication. Can Med Ass J 96: $1112-1113$

18 Sriram K, Schumer W, Ehrenpreis S, Comaty JE, Scheller J (1979) Phenothiazine effect on gastrointestinal tract function. Am J Surg 137: 87-91.

19 Dean GO, Murray JW (1952) Volvulus of the sigmoid colon. Ann Surg 135: 830-840.

20 Ingalls JM, Lynch MF, Schilling JA (1964) Volvulus of the sigmoid in a mental institution. Am J Surg 108: 339-343.

21 Watson N (1981) Late ileus in paraplegia. Paraplegia 19: 13-16.

22 Hoen TI, Cooper IS (1984) Acute abdominal emergencies in paraplegia. Am J Surg 75: 19-24.

23 Miller LS, Staas WE, Herbison GJ (1975) Abdominal problems in patients with spinal cord lesions. Arch Phys Med Rehabil 56: 405-408.

24 Charney KJ, Juler GL, Commar AE (1975) General surgery problems in patients with spinal cord injuries. Arch Surg 110: 1083-1088.

25 Juler GL, Eltorai IM (1985) The acute abdomen in spinal cord injury patients. Paraplegia 23: 118-123.

26 Nay HR, West JP (1967) Treatment of volvulus of the sigmoid colon and caecum. Arch Surg 97: 11-13.

27 Ballantyne GH (1982) Review of sigmoid volvulus: history and results of treatment. Dis Colon Rectum 25: 494-501.

28 Meyers JR, Heifetz CJ, Bave AE (1972) Caecal volvulus. Arch Surg 104: 594-599. 